
DO DIBORETO DE MAGNÉSIO (MgB 2$)$

\title{
THEORETICAL STUDY OF STRUCTURAL GEOMETRY AND ELECTRONIC PROPERTIES OF MAGNESIUM DIBORETH (MgB2)
}

\author{
MATTJE, Vanessa Mendes ${ }^{1}$; PINHEIRO, André Rodrigues²; PEREIRA, Douglas Henrique ${ }^{\star 3}$ \\ 1,2,3 Colegiado de Química, Universidade Federal do Tocantins, Campus de Gurupi, 77410-530, Gurupi - \\ Tocantins, Brasil \\ ${ }^{*}$ Autor correspondente \\ e-mail: doug@uft.edu.br
}

Received 04 August 2018; received in revised form 30 September 02, 07 2018; accepted 03 October 2018

\section{RESUMO}

O presente trabalho fez um estudo teórico molecular das propriedades estruturais e eletrônicas dos isômeros geométricos do diboreto de magnésio $\left(\mathrm{MgB}_{2}\right)$. O sistemas possui características específicas, sendo muito promissor para aplicações tecnológicas, tais como em dispositivos geradores de imagens de ressonância magnética, motores e geradores. O mesmo ainda é empregado nas indústrias de aparelhos eletrônicos, em trens flutuantes (MAGLEV), energia elétrica e no Biomagnetismo. Os resultados demonstram que o isômero geométrico com o menor valor de energia é a espécie cíclica, sendo esta a mais estável. As entalpias padrões de formação encontradas foram de 205,38 Kcal mol-1 e 45,66 Kcal mol-1 para o MgB2-cíclico e para o B-Mg-B respectivamente. Por meio da análise dos orbitais moleculares de fronteira foi possível identificar a forma dos orbitais HOMO - LUMO e como os mesmo contribuem para as bandas ligantes e antiligante e logo para as propriedades supercondutora do composto. Por último, a análise NBO, confirmou que o isômero geométrico mais estável é o $\mathrm{MgB}_{2}$-cíclico, apresentando mais interações entre os orbitais doadores e receptores.

Palavras-chave: $\mathrm{MgB}_{2}$, Energia, Estabilidade, FMO, NBOs

\begin{abstract}
The present work made a molecular theoretical study of the structural and electronic properties of the geometric isomers of magnesium diboride $\left(\mathrm{MgB}_{2}\right)$. The systems have specific characteristics and are very promising for technological applications, such as magnetic resonance imaging devices, motors, and generators. The same is still used in the industries of electronic devices, floating trains (MAGLEV), electricity and Biomagnetism. The results show that the geometric isomer with the lowest energy value is the cyclic species, which is the most stable. The formation enthalpies found were $205.38 \mathrm{Kcal} \mathrm{mol}^{-1}$ and $45.66 \mathrm{Kcal} \mathrm{mol}^{-1} \mathrm{for} \mathrm{MgB}_{2}-$ cyclic and for B-Mg-B respectively. By means of the analysis Frontier Molecular Orbital it was possible to identify the shape of the HOMO - LUMO orbitals and how they contribute to the ligand and anti-ligand bands and thus to the superconducting properties of the compound. Finally, the NBO analysis confirmed that the most stable geometric isomer is MgB2-cyclic, presenting more interactions between donor or receptor orbitals.
\end{abstract}

Keywords: $\mathrm{MgB}_{2}$, Energy, Stability, FMOs, NBOs 


\section{INTRODUÇÃO}

Os estudos de supercondutores têm despertado interesse na comunidade científica, em relação ao seu potencial tecnológico, propriedades físicas e termodinâmicas, como por exemplo: estabilidade energética, condutividade térmica, elásticas, propriedades elétricas e estruturais (Tzeli e Mavridis ,2005 e Lee e Wright, 2004).

Devido à grande importância dos
supercondutores, supercondutor, o diboreto de magnésio $\left(\mathrm{MgB}_{2}\right)$, que possui sua estrutura eletrônica em constante estudo e propriedades supercondutoras excelentes (Hirsch, 2001).

A princípio sintetizaram $0 \mathrm{MgB}_{2}$, um composto intermetálico simples vendido por fornecedores de materiais inorgânicos por aproximadamente três dólares o grama (Branício, 2001). Já a sua composição em forma de pó, a cada dia vêm sendo explorada e estudada com o intuito de uma produção mais rentável e acessível. No entanto, essas propriedades do diboreto de magnésio são peculiares, sendo que ainda estão na fronteira entre a pesquisa e a sua produção industrial (Li e Dou, 2010).

As propriedades do $\mathrm{MgB}_{2}$ fazem dele um material promissor para aplicações tecnológicas, devido apresentar resistividade elétrica nula abaixo de uma temperatura característica. Nessa vertente, quando usado em aparelhos eletroeletrônicos os dispositivos supercondutores proporcionariam a eficiência máxima, favorecendo que toda a energia elétrica seja consumida sem perdas com o efeito Joule. Outras aplicações do diboreto de magnésio são fruto de sua propriedade de diamagnetismo perfeito, fazendo deles essenciais na produção de altos campos magnéticos, podendo ser usado em trens flutuantes (MAGLEV) e até na detecção de campos magnéticos fracos, como por exemplo o Biomagnetismo, que detecta pequenos campos magnéticos gerados pelo corpo humano (CARNEIRO, 2000).

Assim, o $\mathrm{MgB}_{2}$ vêm sendo usado cada vez mais, nas formas de dispositivos geradores de imagens, ressonância magnética, limitadores de corrente, geradores e motores (Tomsic et al., 2007). Com o passar dos anos, houve um interesse em aumentar a densidade da corrente crítica do $\mathrm{MgB}_{2}$, a $4.2 \mathrm{~K}$ e também aumentar a temperatura através da introdução de dopantes (Flükiger e Hossain, 2009). Logo os estudos têm sido otimizados quando se trata da adição de diversas fontes de carbono no bulk do $\mathrm{MgB}_{2}$ (Agatsuma et al.,2006; Yamamoto et al.,2005 e Dou et al., 2002) Com os avanços nas pesquisas em busca de diversas fontes de carbono para uma possível dopagem no $\mathrm{MgB}_{2}$, podemos destacar os compostos: carbono nanométrico (Mudgel et al., 2009), nano SiC e grafeno (De Silva et al., 2012), nanotubos de carbono (Dou et al., 2003), carbeto de sílicio (Dilek et al.,2011; Dou et al., 2003), carboidratos (Kim et al. 2006), carbeto de boro (Zhang et al., 2012), hidrocarbonetos aromáticos (Yamada et al., 2006), grafite (Xu et al., 2004) e diamante (Zhao et al.,2003).

Devido suas inúmeras aplicações trabalhos (Tzeli e Mavridis,2005) vêm sendo realizados com - $\mathrm{MgB}_{2}$ utilizando métodos quânticos como o QCISD/6-311G* e CCSD (T)/cc-pVTZ, em que, os autores prevêem diferentes estados fundamentais para a molécula, como por exemplo, ${ }^{3} B_{1}$ e ${ }^{1} A_{1}$, respectivamente (Tzeli e Mavridis ,2005). Nesse contexto, surgem trabalhos (Lee e Wright, 2004) investigando e obtendo informações espectroscópicas sobre o $\mathrm{MgB}_{2}$. Vale ressaltar que encontrar a estrutura adequada da molécula pode endereçar o sucesso da pesquisa e dos avanços tecnológicos. Além das possibilidades estruturais do $\mathrm{MgB}_{2}$ há também as propriedades eletrônicas dos compostos de Boro como o $\mathrm{MgB}_{2}$ que despertam grande interesse. (Lee e Wright, 2004)

Deste modo, este trabalho teve por objetivo estudar as propriedades eletrônicas e estruturais dos isômeros geométricos da molécula de diboreto de magnésio: $\mathrm{MgBB}, \mathrm{BMgB}, \mathrm{MgB}_{2^{-}}$ cíclico, Figura 1.

a)

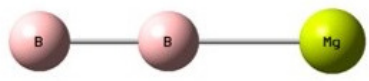

c)

b)
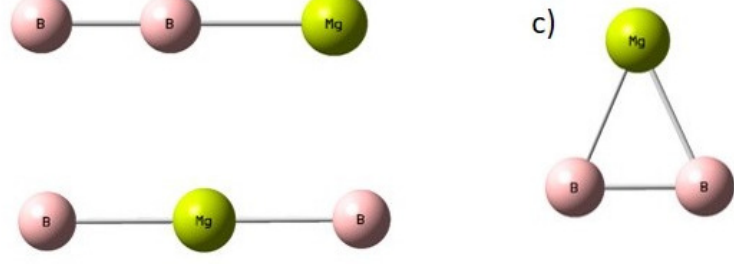

Figura 1. - Representação estrutural dos isômeros do Diboreto de Magnésio: a) MgBB, b) $\mathrm{BMgB}$ e c) $\mathrm{MgB}_{2}$-cíclico. 


\section{MATERIAS E METODOS}

Todas as estruturas foram otimizadas até seu mínimo de energia com o funcional híbrido de três parâmetros de troca e correlação de Becker e Lee, Yang e Parr (Becke, 1988 e Lee 1988), com função de base aug-cc-pVTZ. As frequências vibracionais foram obtidas no mesmo nível de teoria, B3LYP/aug-cc-pVTZ. Cálculos single points foram realizados com a geometria ótima nos níveis MP2, MP3, MP4, MP5 e CCSD(T) com a mesma função de base. Todas as análises mecânico-quânticas realizadas no trabalho usaram a função de onda no nível Coupled-Cluster.

Para avaliar melhor a estabilidade de uma ligação química, utilizou-se o método de Orbitais Naturais de Ligação, NBO - Natural Bond Orbitals (Glendening et al., 2012). A análise permite avaliar a transferência de energia que ocorre de um orbital ligante para um antiligante, incluindo efeitos como de ressonância, eletrônico, estérico, hiperconjugação entre outros (Glendening et al., 2012).

Todos os cálculos foram realizados usando o programa gaussian 09 (Frisch et al.,2009).

\section{RESULTADOS E DISCUSSÃO}

Os resultados do trabalho se encontram divididos nas seguintes seções: geometria estrutural e frequências vibracionais; cálculos de energia; entalpia padrão de formação; orbitais moleculares de fronteira; análises NBOs e considerações finais.

\section{GEOMETRIA ESTRUTURAL E FREQUÊNCIAS VIBRACIONAIS}

Para uma melhor compreensão da molécula de $\mathrm{MgB}_{2}$, foi realizado a elucidação da melhor geometria estrutural dos isômeros. Assim todas as geometrias foram otimizadas e as frequências vibracionais calculadas.

Os resultados dos cálculos das frequências vibracionais harmônicas das moléculas estudadas encontram-se representadas na Tabela 1 . Os resultados evidenciam que 0 isômero $M g-B-B$, apresenta uma frequência negativa com valor de $-136,314$ $\mathrm{cm}^{-1}$ o que demonstra que essa espécie não está no seu mínimo de energia.
Os demais isômeros geométricos não apresentaram frequências imaginárias e somente estes foram consideradas nas etapas conseguintes.

Tabela 1. Frequências encontradas, em $\mathrm{cm}^{-1}$, para as espécies estudadas no trabalho no nível de teoria B3LYP/aug-cc-pVTZ.

\begin{tabular}{lccc} 
Molécula & $\mathbf{1}$ & $\mathbf{2}$ & $\mathbf{3}$ \\
\hline B-Mg-B & 65,885 & 65,885 & 414,009 \\
Mg-B-B & $-136,314$ & 84,114 & 1040,096 \\
$\begin{array}{l}\text { MgB } \\
\text { ciclico }\end{array}$ & 438,122 & 485,209 & $*$ \\
\hline
\end{tabular}

Além do estudo de frequências também foram investigados os comprimentos de ligação para as espécies de interesse, Tabela 2. As moléculas diatômicas $B_{2}$ e MgB foram calculadas para fornecer parâmetros comparativos para os isômeros. Os resultados demonstram que para a espécie B-Mg-B houve um aumento na ligação Mg-B de aproximadamente $0,115 \AA$ quando comparado com a molécula diatômica Mg-B. Já para a espécie cíclica houve uma diminuição de aproximadamente 0,088 Å na ligação $B-B$ e 0,220 $\AA$ para a ligação $\mathrm{Mg}-\mathrm{B}$, quando comparado com as moléculas diatômicas, tal resultado é fruto da formação do anel entre os átomos $\mathrm{Mg}$, B e B, confirme representado na Figura 1c.

Tabela 2. Comprimentos de ligação, em Ångstrom, para os isômeros do diboreto de magnésio e para as espécies $M g B$ e $B_{2}$. Os resultados foram obtidos pelo método B3LYP/ aug-cc-pVTZ.

\begin{tabular}{lcc}
\hline \multicolumn{1}{c}{ Molécula } & Mg-B & B-B \\
\hline $\mathrm{B}-\mathrm{B}$ & - & 1,636 \\
$\mathrm{Mg}-\mathrm{B}$ & 2,423 & - \\
$\mathrm{B}-\mathrm{Mg}-\mathrm{B}$ & 2,538 & - \\
$\mathrm{MgB}_{2}$-cíclico & 2,202 & 1,548 \\
\hline
\end{tabular}

\section{CÁLCULOS DE ENERGIAS}

Os resultados de energia para os métodos B3LYP, MP2, MP3, MP4, MP5 e CCSD (T), estão representados na Tabela 3. Fica evidente que para todos os métodos empregados o isômero geométrico que obteve o menor valor de energia foi o cíclico sendo esse a espécie mais estável. A diferença de energia entre os isômeros é significativa ficando acima de $70 \mathrm{kcal} \mathrm{mol}^{-1}$ para 
todos os métodos.

Tabela 3. Energias, em Hartree, calculadas para os isômeros geométricos $\mathrm{B}-\mathrm{Mg}$ - $\mathrm{B}$ e $\mathrm{MgB} \mathrm{B}_{2}$-cíclico usando os métodos, B3LYP, MP2, MP3, MP4, MP5 e CCSD, com função de base aug-cc-pvTz.

\begin{tabular}{lll}
\hline Método & $\mathrm{B}-\mathrm{Mg}-\mathrm{B}$ & $\mathrm{MgB}_{2}$-cíclico \\
\hline B3LYP & $-249,463779$ & $-249,584002$ \\
MP2 & $-248,825352$ & $-248,970110$ \\
MP3 & $-248,860959$ & $-248,977337$ \\
MP4 & $-248,870487$ & $-248,980732$ \\
MP5 & $-248,881426$ & $-248,999799$ \\
CCSD(T) & $-248,886157$ & $-249,011692$ \\
\hline
\end{tabular}

Entre os métodos ab initio estudados, MP2, MP3, MP4, MP5 e $\operatorname{CCSD}(T)$, o que forneceu a menor energia para os sistemas foi o $\operatorname{CCSD}(T)$. Este resultado é fruto da teoria de Clusteres empregada na metodologia (Morgon, 2007). Os resultados não foram comparados com 0 funcional B3LYP, pois o mesmo se deriva da teoria do funcional de densidade.

Os resultados entrados corroboram com a estrutura cristalina do sólido $\mathrm{MgB}_{2}$ que é composta por triângulos isósceles idênticos como referenciado por Tzeli e Mavridis (2005). É importante ressaltar que cálculos de estados sólidos não foram realizados nesse trabalho e que o enfoque foi puramente molecular

\section{ENTALPIA-PADRÃO DE FORMAÇÃO}

Através da termoquímica do Gaussian foi possível calcular a entalpia de formação, $\Delta_{\mathrm{f}} \mathrm{H}(298,15)$, dos isômeros no nível de teoria $\operatorname{CCSD}(T) /$ aug-cc-pVTZ. Usou-se os valores estabelecidos de $\Delta_{\mathrm{f}} \mathrm{H}(298,15)$ por Ochterski (2000), sendo para o $\mathrm{Mg}\left(34,87 \pm 0,2 \mathrm{Kcal} \mathrm{mol}^{-1}\right)$ e para o $B\left(136,2 \pm 0,2 \mathrm{Kcal} \mathrm{mol}^{-1}\right)$.

A Tabela 4 evidencia os resultados obtidos para as espécies $\mathrm{MgB}_{2}$-cíclico e $\mathrm{B}-\mathrm{Mg}-\mathrm{B}$ e também os resultados referenciados por Lee e Wright (2004) para o isômero cíclico. Os valores de entalpia de formação calculados foram de 205,38 Kcal mol ${ }^{-1}$ para o sistema $\mathrm{MgB}_{2}$-cíclico e de $45,66 \mathrm{Kcal} \mathrm{mol}^{-1}$ para o B-Mg-B. Comparando o resultado encontrado de entalpia de formação para o isômero cíclico com os da literatura, podese verificar uma pequena diferença quando comparados resultado das metodologias adotas. É importante citar que não foram encontrados valores experimentais de entalpia de formação das espécies estudadas e por isso não houve comparação com os valores experimentais. Os resultados calculados também podem ser usado como referência para outros estudos visto que o nível utilizado é no trabalho é de alta acuracidade.

Tabela 4. Entalpia de formação $\left(\Delta_{f} \mathrm{H}\right.$ em Kcal mol $\left.{ }^{1}\right)$ para os isômeros geométricos $\mathrm{MgB}{ }_{2}$ cíclico e $B$ $M g-B$.

\begin{tabular}{lcc}
\hline Molécula & $\Delta_{\mathrm{f}} \mathbf{H}(\mathbf{2 9 8 , 1 5 )}$ & $\begin{array}{c}\Delta_{\mathrm{f}} \mathbf{H}(\mathbf{2 9 8 , 1 5}) \\
(\mathbf{a})\end{array}$ \\
\hline B-Mg-B & 45,66 & - \\
$\mathrm{MgB}_{2}$ - cíclico & 205,38 & $194,3 \pm 0,2$ \\
\hline
\end{tabular}

Nota: (a) Dados obtidos a partir da referência Lee e Wright (2004).

\section{ORBITAIS FRONTEIRA}

MOLECULARES

DE

Com a intenção de elucidar a reatividade química em nível atômico, surgiram os orbitais moleculares de fronteira (FMO: Frontier Molecular Orbital). Através desse conceito foi possível predizer a posição mais reativa sobre as espécies químicas, sendo capaz de analisar a densidade eletrônica através dos orbitais das moléculas (Pereira et al., 2016).

Segundo Fukui, o HOMO ou o LUMO podem não ser adequados para uma dada reação, mas, certamente o próximo orbital ou qualquer orbital que tem energia muito próxima do HOMO ou do LUMO poderia ser usado (Pereira et al., 2016). Tendo em vista a grande importância dos orbitais de fronteira, cálculos dos mesmos foram realizados paras as espécies $\mathrm{MgB}_{2}$-cíclico e $\mathrm{B}$ Mg-B, Figura 2.
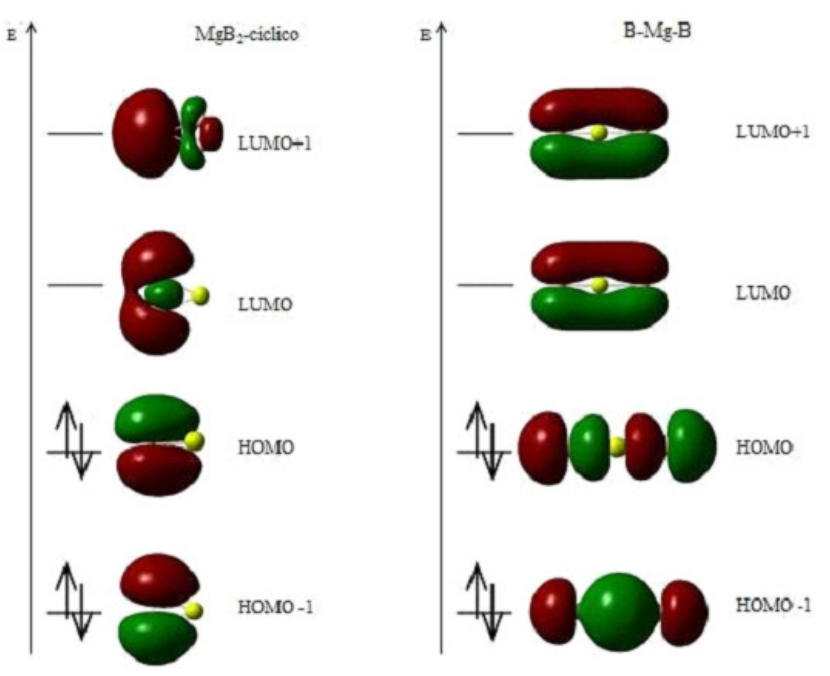

Figura 2. - Orbitais Moleculares de Fronteira para a) MgB2-Ciclico e b) $B-M g-B$. 
Através da Figura 2, observa-se que os isômeros $\mathrm{MgB}_{2}$-cíclico e $\mathrm{B}-\mathrm{Mg}-\mathrm{B}$ apresentam o orbital HOMO no formato $\pi$ no plano da molécula.

A forma dos orbitais evidencia a formação de bandas $\pi$ ligante e também anti-ligantes. Uma possível explicação para esse composto apresentar propriedades de supercondução são devido suas bandas que estão ligadas em camadas de átomos de boro adjacentes, em que a sua densidade de carga estende-se nas direções paralelas e também nas direções perpendiculares aos planos dos átomos de Boro, favorecendo a origem de estados de condução tridimensionais. A Figura 2 mostra a o formato dos orbitais e essa tendência.

Além disso, é necessário levar em consideração o potencial atrativo dos íons $\mathrm{Mg}^{2+}$, que favorece a transferência de cargas das entre as bandas.

\section{ORBITAIS NATURAIS DE LIGAÇÃO ( NBO)}

Foi realizada a análise dos orbitais naturais de ligação, NBOs, com a finalidade de avaliar as principais interações estabilizadoras que ocorrem entre os NBOs formalmente ocupados e desocupados.

Nas tabelas 5-7 estão representadas as principais interações para as moléculas $\mathrm{B}_{2}, \mathrm{~B}-\mathrm{Mg}$ $\mathrm{B}$ e $\mathrm{MgB}_{2}$-cíclico.

Tabela 5. Energia de estabilização de segunda ordem $\left(\Delta E^{2}\right.$ em kcal. $\left.\mathrm{mol}^{-1}\right)$, diferenças de energia entre orbitais $\left(\varepsilon_{i}-\varepsilon_{j}\right)$, e elementos da matriz de Fock $(F(i, j))$, do $B_{2}$.

\begin{tabular}{ccccc}
$\begin{array}{c}\text { NBO } \\
\text { doador }\end{array}$ & $\begin{array}{c}\text { NBO } \\
\text { receptor }\end{array}$ & $\begin{array}{c}\Delta \mathbf{E}^{2} \\
\mathbf{k c a l . m o l}\end{array}$ & $\left(\varepsilon_{\mathbf{i}}-\varepsilon_{\mathrm{j}}\right)$ & $\begin{array}{c}\mathbf{F}(\mathbf{i}, \mathbf{j}) \\
\mathbf{u . a}\end{array}$ \\
\hline$\sigma \mathrm{B} 1-\mathrm{B} 2$ & $\pi^{*} \mathrm{~B} 1-\mathrm{B} 2$ & 150,57 & 0,55 & 0,256 \\
$\pi \mathrm{B} 1-\mathrm{B} 2$ & $\mathrm{n} \sigma^{*} \mathrm{~B} 1$ & 5,96 & 0,77 & 0,065 \\
$\pi \mathrm{B} 1-\mathrm{B} 2$ & $\mathrm{n} \sigma^{*} \mathrm{~B} 2$ & 5,33 & 0,77 & 0,061 \\
$\pi \mathrm{B} 1-\mathrm{B} 2$ & $\sigma^{*} \mathrm{~B} 1-\mathrm{B} 2$ & 165,54 & 0,64 & 0,291 \\
$\pi \mathrm{B} 1-\mathrm{B} 2$ & $\pi^{*} \mathrm{~B} 1-\mathrm{B} 2$ & 11,42 & 0,22 & 0,045 \\
\hline
\end{tabular}


Para a espécie $\mathrm{Mg}-\mathrm{B}$ os resultados não aparecem representados, pois não houve nenhuma interação significativa entre os orbitais, acima de $3 \mathrm{kcal} \mathrm{mol}^{-1}\left(\Delta \mathrm{E}^{2}\right)$.

Os resultados de NBO confirmam que o isômero geométrico mais estável é o $\mathrm{MgB}_{2}$-cíclico, uma vez que ele apresenta um maior número de interações entre o NBO doador e receptor e energias de estabilização superiores e mais significativas do que os outros isômeros.

O resultado de NBO vão de encontro com os resultados anteriores obtidos.

\section{CONCLUSÕES}

O trabalho evidenciou que entre os isômeros estudados o mais estável é $\circ \mathrm{MgB}_{2}-$ cíclico, pois é a espécie que apresentou o menor valor de energia para todos os métodos empregados.

As entalpias de formação encontrada para os isômeros $\mathrm{MgB}_{2}$-cíclico e $\mathrm{B}-\mathrm{Mg}$ - $\mathrm{B}$ foram de $205,38 \mathrm{Kcal} \mathrm{mol}^{-1}$ e $45,66 \mathrm{Kcal}_{\mathrm{mol}^{-1}}$ respectivamente. Os resultados de entalpia foram calculados em nível CCSD(T)/aug-cc-pVTZ e podem ser utilizados como referências, pois não foi encontrados valores experimentais para as espécies. Já Por meio da análise dos orbitais moleculares de fronteira foi possível identificar a forma dos orbitais HOMO e LUMO, além de aferir a forma das bandas ligantes e antiligante. Por fim através da análise NBO, verificou-se que o isômero $\mathrm{MgB}_{2}$-cíclico apresenta mais interações entre os orbitais doadores e receptores estabilizando assim e estrutura.

\section{AGRADECIMENTOS}

O autor D. H. Pereira agradece o suporte financeiro do $\mathrm{CNPq}$ (Conselho Nacional de Desenvolvimento Científico e Tecnológico, processo n. 446651/2014-1), a CAPES e a Universidade Federal do Tocantins-UFT, pois 0 desenvolvimento desta pesquisa contou com benefícios do Programa Novos Pesquisadores da UFT (PROPESQ/UFT). O autor também agradece o Centro Nacional de Processamento de alto Desempenho em São Paulo CENAPAD e a UNICAMP pelos recursos computacionais.

\section{REFERÊNCIAS:}

1. Agatsuma, K.; Furuse, M.; Umeda, M.; Fuchimo, S.; Lee, W. J.; Hur, J. M. Trans. Appl. Supercond, 2006, 16, 1407.

2. Becke, A. D. Phys. Rev. A 38, 1988, 3098.

3. Branício, P. S. Rev. Bras. Ensino Fis, 2001, 23,381.

4. Carneiro, A. A. O.; Ferreira, A.; Moraes, E. R.; Araújo, D. B.; Sosa, M.; Baffa, O.; Rev. Bras. Ensino Fis, 2000, 22, 324.

5. De Silva, K. S. B. Scripta Mater, 2012, 67, 802.

6. Dilek, T. J. Supercond. Nov. Magn, 2011, $24,495$.

7. Dou, S. X. Appl. Phys. Lett, 2002, 81,3419.

8. Dou, S.X. J. Appl. Phys, 2003, 94, 1850.

9. Flükiger R.; Hossain, M. S. A.; Senatore C, 2009, Supercond. Sci. Tech, 22,095016.

10. Glendening, E.D.; Landis, C.R.; Weinhold, F. Rev. Comput. Mol. Sci, 2012, 2, 1.

11. Hirsch, J. E. Phys. Lett. A, 2001, 282,392.

12. Kovác, P.; Husek, I.; Melišek, T.; Kopera, L.; Reissner, M. Supercond. Sci. Tech, 2010, 23,1.065010.

13. Kim, J. H.; Zhou, S.; Hossain, M. S. A.; Pan, A. V. e Dou, S. X. Appl. Phys. Lett, 2006, 89,142505.

14. Kitaguchi, H.; Kumakura, H.; and Togano, K. Phys. C. 2001, 363

15. Kovác,P.; Dhallé'M.; Melišek,T.; van Eck, H J N; Wessel, W A J; ten Haken, B; Hušek, I. Supercond. Sci. Technol, 2003, 16.

16. Lee, C.; Yang, W. and Parr, R. G. Phys. Rev. B, 1988, 37, 785.

17. Lee, E. P. F.; Wright, T. G. J. Phys. Chem, 2004, 108, 7424.

18. Li, W.; Dou, S. X. Superconducting properties of carbonaceous chemical doped $\mathrm{MgB}_{2}$ Superconductor. ed A M Luiz (Rijeka, Croatia: InTech), 2010, chapter 6.

19. Frisch, M. J.; Trucks, G. W.; Schlegel, H. B.; Scuseria, G. E.; Robb, M. A.; Cheeseman, J. R.; Scalmani, G.; Barone, V.; Mennucci, B.; Petersson, G.A.; Nakatsuji, H.; Caricato, M.; Li, X.; Hratchian, H. P.; Izmaylov, A. F.; Bloino, J.; Zheng, G.; Sonnenberg, J. L.; Hada, M.; Ehara, M.; Toyota, K.; Fukuda, R.; Hasegawa, J.; Ishida, M.; Nakajima, T.; Honda, Y.; Kitao, O.; Nakai, H.; Vreven, T.; Montgomery, J. A.; Peralta Jr., J. E.; Ogliaro, F.; Bearpark, M.; Heyd, J. J.; Brothers, E.; Kudin, K. N.; Staroverov, V. 
N.; Kobayashi, R.; Normand, J.; Raghavachari, K.; Rendell, A.; Burant, J. C.; lyengar, S. S.; Tomasi, J.; Cossi, M.; Rega, N.; Millam, J. M.; Klene, M.; Knox, J. E.; Cross, J. B.; Bakken, V.; Adamo, C.; Jaramillo, J.; Gomperts, R.; Stratmann, R. E.; Yazyev, O.; Austin, A. J.; Cammi, R.; Pomelli, C.; Ochterski, J. W.; Martin, R. L.; Morokuma, K.; Zakrzewski, V. G.; Voth, G. A.; Salvador, P.; Dannenberg, J. J.; Dapprich, S.; Daniels, A. D.; Farkas, Ö.; Foresman, J. B.; Ortiz, J. V.; Cioslowski, J.; Fox, D. J.; Gaussian 09, Revision D.01, Gaussian, Inc.: Wallingford CT, 2009.

20. Morgon, N. H.; Coutinho, K. Métodos de química teórica e modelagem molecular. São Paulo: Editora Livraria da Física, 2007.

21. Mudgel, M.; Chandra,V.; Ganesan, L. S. S.; Bhalla, G. L.; Kishan, H. and Awana, V. P. S. J. Appl. Phys, 2009, 106, 033904.

22. Ochterski, J. W. Thermochemistry in Gaussian, 2000, 19, acessado em janeiro de 2018.

23. Pereira, D. H.; Porta, F. A. L. ; Santiago, R. ; Garcia, D. R.; Ramalho, T. C. Rev. Virt. Quím, 2016, 8, 425

24. Tomsic, M.; Rindfleisch, M.; Yue, J.J; McFadden, K.; Phillips, J.; Sumption, M. D.; Bhatia, M., Bohnenstiehl, S. D.; and Collings, E. W. Int. J. Appl. Ceram. Technol. 2007, 4, 250.

25. Tzeli,D., Mavridis, A. J. Phys. Chem, 2005, 109, 10663.

26. Xu, H. L. China Phys. Lett, 2004, 21, 2511.

27. Yamada, H.; Hirakawa, M.; Kumakura $\mathrm{H}_{\text {.; }}$ and Kitaguchi, H. Supercond. Sci. Tech.y. 2006, 19, 175.

28. Yamamoto, A.; Shimoyama, J.; Ueda, S.; Iwayama, I.; Horii, S. and Kishio, K. Supercond. Sci. Tech, 2005,18, 1323.

29. Yamamoto, A.; Shimoyama, J.; Ueda, S.; Katsura, Y.; Iwayama, I.; Horii, S. and Kishio K. Appl. Phys. Lett, 2005, 86,1.937991.

30. Zhang, Y. B.; Shan, X.J.; Bai,X. W.; Liu, T.Y.; Zhu, H. M. and Cai, C.B. Supercond. Sci. Tech, 2012, 25,095003.

31. Zhao, Y.; Cheng, C.H. Appl. Phys. Lett, 2003, 83,1.1606884.

PERIÓDICO TCHÊ QUÍMICA • www.periodico.tchequimica.com • Vol. 16 N. 31 • ISSN 1806-0374 (impresso) • ISSN 1806-9827 (CD-ROM) • ISSN 2179-0302 (meio eletrônico) (C) 2019. Porto Alegre, RS. Brasil

OPEN ACCESS. This article is licensed under a Creative Commons Attribution 4.0 (CC BY 4.0) International License, which permits use, sharing, adaptation, distribution, and reproduction in any medium or format, as long as you give appropriate credit to the original author(s) and the source, provide a link to the Creative Commons license, and indicate if changes were made. The images or other third-party material in this article are included in the article 's Creative Commons license unless indicated otherwise in a credit line to the material. If material is not included in the article's Creative Commons license and your intended use is not permitted by statutory regulation or exceeds the permitted use, you will need to obtain permission directly from the copyright holder. To view a copy of this license, visit http://creativecommons.org/licenses/by/4.0/. 